\title{
1 Comparison of starch granule sizes of maize seeds between parental lines
}

\section{2 and derived hybrids}

3 Running head: Starch granule sizes in maize parental and hybrid seeds

$4 \quad$ Liangjie Liu, Wei Wang*

5 National Key Laboratory of Wheat and Maize Crop Science, College of Life Sciences, Henan

6 Agricultural University, Zhengzhou 450002, China

\section{Correspondence}

8 Prof. Wei Wang; email: wangwei@ henau.edu.cn 


\section{Abstract}

10 Maize starch is an important agricultural commodity that serves as food, feed, and a raw

11 material for industrial purposes. It is organized into starch granules (SG) inside amyloplasts

12 and is highly accumulated in endosperms. Maize hybrids exhibit heterosis over their parents.

13 However, the parental effect on the size of SG in F1 hybrid seeds remains unclear. Here we

14 compared the seed SG sizes among two parental inbred lines (Chang7-2 and Zheng58) as well

15 as their reciprocal hybrids. SG was observed in situ and in vitro with SEM. The size of seed

16 SG in hybrids was more like that of female parents, especially for large SG population. Thus,

17 the control of SG size exhibits a maternal inheritance trend in the context of plastid

18 (amyloplast) inheritance. Our results provide some insight on selecting parental inbred lines

19 for breeding maize hybrids with different SG sizes.

20 Keywords: maize seeds, hybridization, starch granule size, maternal effect, microscopy

21 Abbreviations: SG, starch granules. 


\section{Introduction}

Starch is the predominant carbohydrate reserve in plants (Zeeman et al. 2010). It consists of glucose residues linked to each other by $\alpha$-1,4-linkages (amylose) with occasional $\alpha$-1,6-branches (amylopectin) (Zeeman et al. 2010). In addition to as food and feed, starch is used as texturizer, gelling agent, thickener, adhesive, moisture-retainer, biofuels etc. (Niu et al. 2019).

Starch accumulates in amyloplasts in a semi-crystalline granular form, which is divided into two forms: storage starch and transient starch (Zeeman et al. 2010). Storage starch granule (SG) mainly exists in cereal seeds, storage roots, and tubers, with a highly varied size of 2 to $50 \mu \mathrm{m}$ dependent on plant species (Seung and Smith 2019). Transient SG is accumulated in leaf chloroplast and is usually small $(\sim 2 \mu \mathrm{m})$ with a uniformly flattened disc shape among plant species (Seung et al. 2017, Yu et al. 2017, Zeeman et al. 2002). The SG with different sizes can be used for specific applications. Large SG with more amylose, higher peak, trough and final pasting viscosities, and lower gelatinization temperature than small SG (Cai et al. 2014), resulting in different bread- and noodle-making quality ( $\mathrm{Zi}$ et al. 2019). Fine SG can be used for generating thin films (Lindeboom et al. 2004) and fatty mouthfeel (Ma et al. 2006).

Many studies have demonstrated that the morphology and size of storage SG are mainly determined by species origin (Zeeman et al. 2010), biochemistry and physiology of plants (Gregorová et al. 2006, Alcázar-Alay and Meireles 2015, Zhao et al. 2018). Some QTLs and protein (e.g. SUBSTANDARD STARCH GRAIN6, starch synthase I) controls SG size have been identified in maize kernels, rice and wheat endosperm using mutant (Liu et al. 2018, Matsushima et al. 2016, McMaugh et al. 2014). However, little reports about the effect of parental inbred lines on the seed SG size in derived hybrids in maize. 
Maize (Zea mays) is globally grown for its high seed starch content. Its hybrids exhibit heterosis over parental lines in plant growth and development, yield and stress tolerance (Hoecker et al. 2006). The hybrid kernel traits, especially weight and size, are mostly affected by female parents (Zhang et al. 2016). Here two inbred lines Chang7-2 (C7-2) and Zheng58 (Z58) and their derived hybrids were used to instigate the parental effect on the SG sizes by scanning electron microscopy.

\section{Materials and methods}

Maize inbred lines C7-2 and Z58 were grown and reciprocally crossed as described previously (Ning et al. 2017). Mature seeds of C7-2, Z58, C7-2 $\times$ Z58, and Z58 $\times$ C7-2 were used here. Scanning electron microscopy (SEM) observation of seed SG in situ and in vitro was exactly performed as our recent work (Niu et al. 2019). The SG size was expressed as spherical diameter by measuring at least 300 granules. All assays were conducted in at least three biological replicates. Statistically significant differences were accessed by the Student's t-test $(\mathrm{p}<0.05)$.

\section{Results}

The seeds of C7-2 and Z58 significantly differ in shape, size and weight. The C7-2 seeds were like triangle and longer, whereas the Z58 seeds were rounder and wider (Fig. S1,a-c). The derived hybrids were like the female parent in morphology but with an increased size and weight (Fig. S1, d-e). Moreover, the proportion of the endosperm in the hybrid seeds was determined by the female parent (Fig. S1,f).

The seed SG in situ of the four materials were in spherical shape (Fig. 1a, Fig. S2), but the SG surface of the parental seeds was smooth but was rough in the hybrids with more membrane debris. The size of SG in all samples ranged from 4.5 to $19.5 \mu \mathrm{m}$, and the hybrid seeds were more similar to the female parent in the average SG size and distribution (Fig. 
1b-1c), especially the ratio of large SG population (Fig. 1d), exhibiting a maternal inheritance trend.

The seed SG in vitro in all samples were mostly polygonal with similar surface features, because the membrane debris on the surface of the SG were removed during the isolation (Fig. 2a). The size of the isolated SG varied from 4.7 to $27.98 \mu \mathrm{m}$ (Fig. 2b), greater than that in situ. Even so, the size distribution of the SG in vitro was comparable to the in vivo results (Fig. 2d).

Additionally, the SG size correlated with the 100-kernel weight and the endosperm sizes, with correlation coefficients of $0.5595(\mathrm{p}<0.05)$ and $0.5773(\mathrm{p}<0.05)$, respectively (Fig. S3).

\section{Discussion}

Maize seeds consist of up to $78 \%$ starch that is overwhelmingly accumulated in the endosperm. The endosperm plays a key role in determining seed size (Berger et al. 2006). Large SG is the major determinant of wheat endosperm weight (Chojkecki et al. 1986). Female parent significantly influences maize hybrid seed traits through altering endosperm cell number (Jones et al. 1996).

In the present study we found the SG size in hybrid seeds was more affect by the female parent. Two reasons may explain this phenomenon. First, the endosperm is a triploid tissue that receives two genomes from mother and one genome from the paternal side $(\mathrm{Li}$ and $\mathrm{Li}$ 2015). Gómez et al. (2002) found endosperm transfer cell can facilitate the transport of maternal solutes and nutrients at the interface between maternal tissues and the endosperm. Second, starch is synthesized and stored in plastids (chloroplasts/amyloplasts) in leaves or seeds (Seung and Smith 2019). Plastids are maternally inherited in plants, as observed in Epilobium plants by electron microscopy (Schmitz and Kowallik 1987). It is suggested that maternal plastid inheritance is the elimination of paternal plastids during fertilization or the exclusion of plastids from germ cells during pollen development (Kuroiwa 2010). However, 
95

the mechanisms of parental inbred lines on the SG size in their hybrid seeds remains unclear in the case of maize.

The size and shape of the SG are controlled by multiple factors, such as botanical species origin, biochemistry and physiology of plants, and SG properties (Ellis et al. 1998, Nordmark and Ziegler. 2002, Ji et al. 2003a, 2003b, Lu et al. 2011, Cai et al. 2014). Super-sweet, pop, waxy, and dent maize differ significantly in size and shape of seed SG (Cui et al. 2014). The SG size and morphology in maize seeds are affected by nitrogen levels (Zhao et al. 2018). A significant SNPs within genes GRMZM2G419655 and GRMZM2G511067 was identified as functional markers in screening maize SG size (Liu et al. 2018). In rice, amyloplast membrane protein SUBSTANDARD STARCH GRAIN6 can control SG size in endosperm (Matsushima et al. 2016). In wheat, starch synthase I can change the morphology, size and fine structure of starch in wheat endosperm by affecting the amylose content (McMaugh et al. 2014). However, the mechanism controlling the size and shape is largely unclear.

It is needed to note that maize seed SG were generally spherical in situ with and polygonal in vitro with comparative sizes as observed previously ( $\mathrm{Li}$ et al. 2007, Zhang et al. 2011, Paraginski et al. 2014, Zhao et al. 2018, Niu et al. 2019). The large SG took a slight greater proportion in the isolated SG, possibly because some of the fine SG was lost during the isolation and all SG may somewhat swell from water-absorbing during the isolation. In addition, only two elite inbred lines C7-2 and Z58 in China were used in the present study and their SG size was different but comparable, if the SG size of the parental lines were greatly varied, the derived hybrids may varied greatly in seed SG sizes.

We found that the size of seed SG in hybrids was more like that of female parents, especially for large SG population, exhibiting a maternal inheritance trend. This provides some insight on selecting parental inbred lines for breeding maize hybrids with different SG sizes for different applications. 


\section{References}

Alcázar-Alay, S.C., Meireles, M.A.A. 2015. Physicochemical properties, modifications and applications of starches from different botanical sources. Journal of Food Science and Technology -Mysore 35, 215-236.

Berger, F., Grini, P.E., Schnittger, A., 2006. Endosperm: an integrator of seed growth and development. Current Opinion in Plant Biology 9, 664-670.

Cai, C.H., Lin, L.S., Man, J.M., Zhao, L.X., Wang, Z.F., Wei, C.X. 2014. Different structural properties of high-amylose maize starch fractions varying in granule size. Journal of Agricultural and Food Chemistry 62, 11711-11721.

Chojkecki, A.J.S., Gale, M.D., Bayliss, M.W. 1986. The number and sizes of starch granules in the wheat endosperm, and their association with grain weight. Annals of Botany 58, 819-831.

Cui, L., Dong, S., Zhang, J., Liu, P. 2014. Starch granule size distribution and morphogenesis in maize (Zea mays L.) grains with different endosperm types. Australian Journal of Crop Science 8, 1560-1565.

Ellis, R.P., Cochrane, M.P., Dale, M.F.B., Duffus, C.M., Lynn, A., Morrison, I.M., Prentice, R.D.M., Swanston, J. S., Tiller S.A. 1998. Starch production and industrial use. Journal of the Science of Food and Agriculture 77, 289-311.

Gregorová, E., Pabst, W., Bohačenko, I. 2006. Characterization of different starch types for their application in ceramic processing. Journal of the European Ceramic Society 26, 1301-1309.

Hoecker, N., Keller, B., Piepho, H.P., Hochholdinger, F. 2006. Manifestation of heterosis during early maize (Zea mays L.) root development. Theoretical and Applied Genetics 112, 421-429.

Ji, Y., Seetharaman, K., Wong, K., Pollak, L.M., Duvick, S., Jane, J., White, P.J. 2003a. Thermal and structural properties of unusual starches from developmental corn lines. Carbohydrate Polymers 51: 439-450.

Ji, Y., Wong, K., Hasjim, J., Pollak, L.M., Duvick, S., Jane, J., White, P.J. 2003b. Structure and function of starch from advanced generations of new corn lines. Carbohydrate Polymers 54,305-319.

Jones, R.J., Schreiber, B.M.N., Roessler, J.A. 1996. Kernel sink capacity in maize: genotypic and maternal regulation. Crop Science 36, 301-306. 
152

153

154

155

156

157

158

159

160

161

162

163

164

165

166

167

168

169

170

171

Kuroiwa, T. 2010. Review of cytological studies on cellular and molecular mechanisms of uniparental (maternal or paternal) inheritance of plastid and mitochondrial genomes induced by active digestion of organelle nuclei (nucleoids). Journal of Plant Research 123, 207-230.

Li, L., Blanco, M., Jane, J.L. 2007. Physicochemical properties of endosperm and pericarp starches during maize development. Carbohydrate Polymers 67: 630-639.

Li, N., Li, Y. 2015. Maternal control of seed size in plants. J Exp Bot. 66(4): 1087-1097.

Lindeboom, N., Chang, P.R., Tyler, R.T. 2004. Analytical, biochemical and physicochemical aspects of starch granule size, with emphasis on small granule starches: a review. Starch/Stärke. 56: 89-99.

Liu, N., Zhang, Z., Xue, Y., Meng, S., Huang, Y., Li, W., Huang, J., Tang, J. 2018. Identification of quantitative trait loci and candidate genes for maize starch granule size through association mapping. Scientific Reports 8, 14236.

Lu, D.L., Guo, H.F., Lu, W.P. 2011. Effects of sowing date, variety and nitrogen top-dressing at jointing stage on starch granule size distribution of waxy maize. Acta Agronomica Sinica 44, 263-270.

Ma, Y., Cai, C., Wang, J., Sun, D.W. 2006. Enzymatic hydrolysis of corn starch for producing fat mimetics. Journal of Food Engineering 73, 297-303.

Matsushima, R., Maekawa, M., Kusano, M., Tomita, K., Kondo, H., Nishimura, H., Crofts, N., Fujita, N., Sakamoto, W. 2016. Amyloplast membrane protein SUBSTANDARD STARCH GRAIN6 controls starch grain size in rice endosperm. Plant Physiology 170, 1445-1459.

McMaugh, S.J., Thistleton, J.L., Anschaw, E., Luo, J., Konik-Rose, C., Wang, H., Huang, M., Larroque, O., Regina, A., Jobling, S.A., Morell, M.K., Li, Z. 2014. Suppression of starch synthase I expression affects the granule morphology and granule size and fine structure of starch in wheat endosperm. Journal of Experimental Botany 65, 2189-2201.

Ning, F., Wu, X., Zhang, H., Wu, Z., Niu, L., Yang, Hao., Wang, W. 2017. Accumulation profiles of embryonic salt-soluble proteins in maize hybrids and parental lines indicate matroclinous inheritance: A proteomic analysis. Frontiers in Plant Science 8: 1824.

Niu, L., Ding, H., Hao, R., Liu, H., Wu, X., Hu, X., Wang, W. 2019. A rapid and universal method for isolating starch granules in plant tissues. Plant Cell and Environment 42, 3355-371.

Nordmark, T.S., Ziegler, G.R. 2002. Structural features of non-granular spherulitic maize starch. Carbohydrate Research 337, 1467-1475. 
185

186

187

188

189

190

191

192

193

194

195

196

197

198

199

200

201

202

203

204

205

206

207

208

209

210

211

Paraginski, R.T., Vanier, N.L., Moomand, K., de Oliveira, M., Zavareze Eda, R., Marques e Silva, R., Ferreira, C.D., Elias, M.C. 2014. Characteristics of starch isolated from maize as a function of grain storage temperature. Carbohydrate Polymers 102, 88-94.

Seung, D., Boudet, J., Monroe, J., Schreier, T.B., David, L. C., Abt, M., Lu, K.J., Zanella, M., Zeeman, S.C. 2017. Homologs of PROTEIN TARGETING TO STARCH control starch granule initiation in Arabidopsis leaves. Plant Cell, 29, 1657-1677.

Seung, D., Smith, A.M. 2019. Starch granule initiation and morphogenesis-progress in Arabidopsis and cereals. Journal of Experimental Botany 70, 771-784.

Yu, S., Zhang, F., Li, C., Gilbert, R. G. 2017. Molecular structural differences between maize leaf and endosperm starches. Carbohydrate Polymers 161: 10-15.

Zeeman, S. C., Tiessen, A., Pilling, E., Kato, K. L., Donald, A. M., Smith, A.M. 2002. Starch synthesis in Arabidopsis. Granule synthesis, composition, and structure. Plant Physiology 129, 516-529.

Zeeman, S.C., Kossmann, J., Smith, A.M., 2010. Starch: its metabolism, evolution, and biotechnological modification in plants. Annual Review of Plant Biology 61, 209-234.

Zhang, L., Zhang, J.W., Liu, P., Dong, S.T. 2011. Starch granule size distribution in grains of maize with different starch contents. Scientia Agricultura Sinica 44: 1596-1602.

Zhang, X.D., Chen, Y.F., Zhang, R.H., Zhong, Y.Y., Luo, Y., Xu, S.T., Liu, J.C., Xue, J.Q., Guo, D.W. 2016. Effects of extrusion treatment on physicochemical properties and in vitro digestion of pregelatinized high amylose maize flour. Journal of Cereal Science 68, 108-115.

Zhao, F., Jing, L., Wang, D., Bao, F., Lu, W., Wang, G. 2018. Grain and starch granule morphology in superior and inferior kernels of maize in response to nitrogen. Scientific Reports 8: 6343.

Zi, Y., Shen, H., Dai, S., Ma, X., Ju, W. 2019. Comparison of starch physicochemical properties of wheat cultivars differing in bread- and noodle-making quality. Food Hydrocolloids 93, 78-86. 


\section{Figure Legends}

213 Fig. 1. The morphology and size of seed SG in situ. a SEM observation. Bar $=20 \mu \mathrm{m}$. b

214 Average SG diameter of. c SG size distribution. d The size change in different SG 215 populations.

216 Fig. 2. The morphology and size of seed SG in vitro. a SEM observation. Bar $=20 \mu \mathrm{m}$. b 217 Average SG diameter of. c SG size distribution. d The size change in different SG.

\section{Supporting Information}

219 Fig. S1. Phenotypic analysis of seeds. a Seeds of two inbred lines and their reciprocal hybrids.

220 Bar $=1 \mathrm{~cm}$. b The length of kernels (mean $\pm \mathrm{SD}, \mathrm{n}=50)$. $\mathbf{c}$ The width of kernels (mean $\pm \mathrm{SD}$, $221 \mathrm{n}=50)$. d The 10-kernel volume (mean $\pm \mathrm{SD}, \mathrm{n}=5$ ). e The 100-grain weight (mean $\pm \mathrm{SD}$, $\mathrm{n}=5$ ). $\mathbf{f}$ The ratio of endosperm in seed (mean $\pm S D, n=5)$.

Fig. S2. Comparison of the morphology and size of seed SG between two maize inbred lines and derived hybrids by SEM observation in situ. Bar $=20 \mu \mathrm{m}$.

225 Fig. S3. Correlation analysis of SG size, seed weight and endosperm size between two maize 226 inbred lines and derived hybrids. 
$\mathbf{a}$

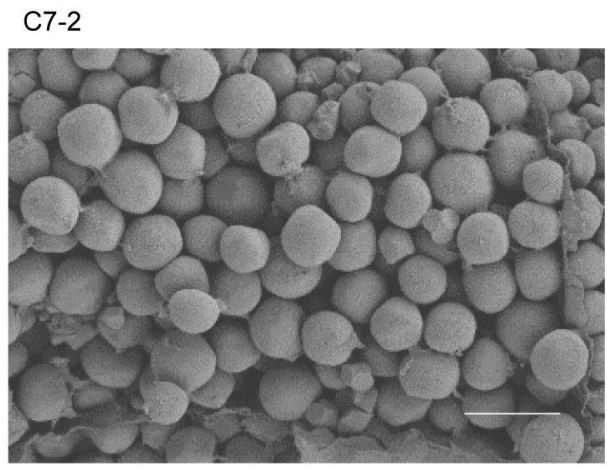

C7- $2 \times Z 58$
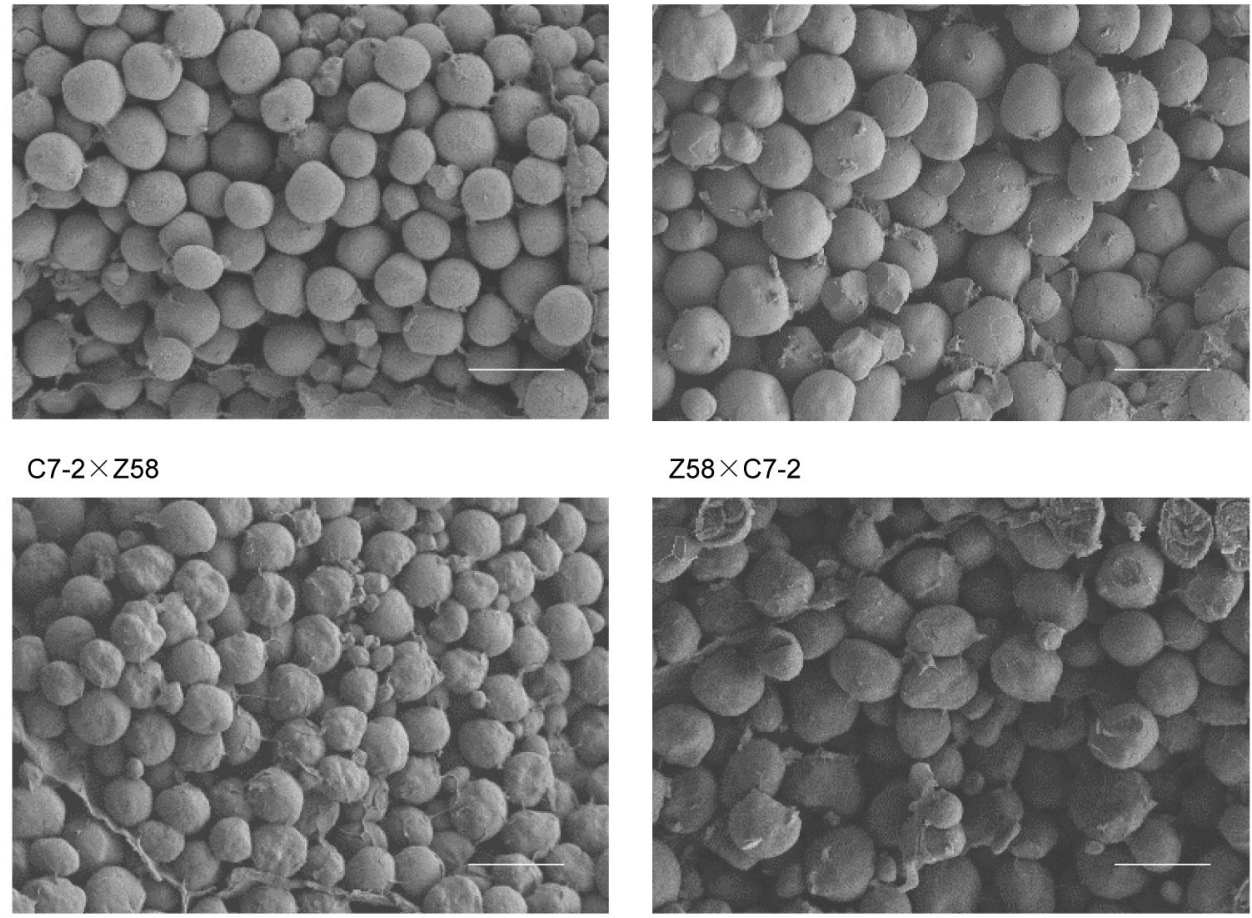

\section{$\mathrm{Z} 58 \times \mathrm{C} 7-2$}

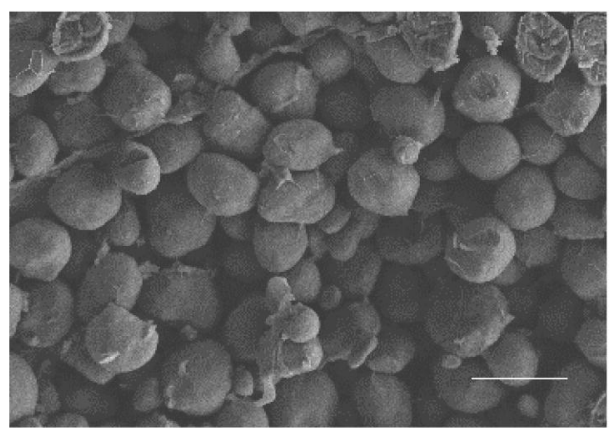

C

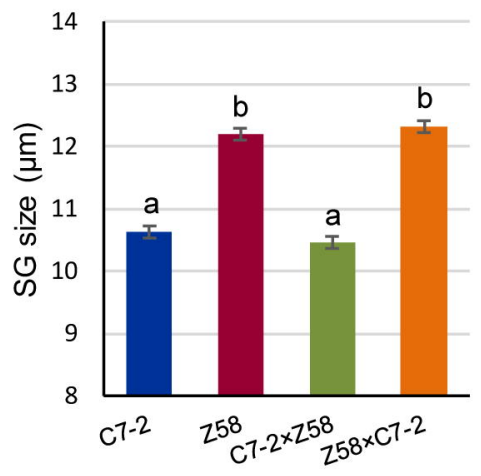

d

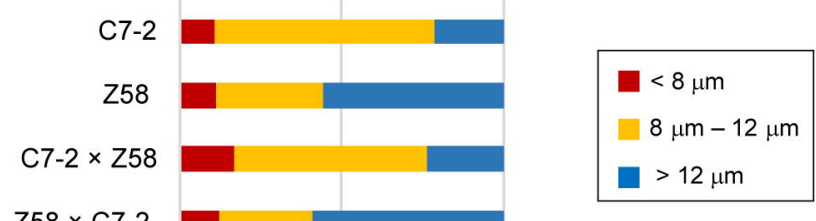

$\mathrm{Z} 58 \times \mathrm{C} 7-2$

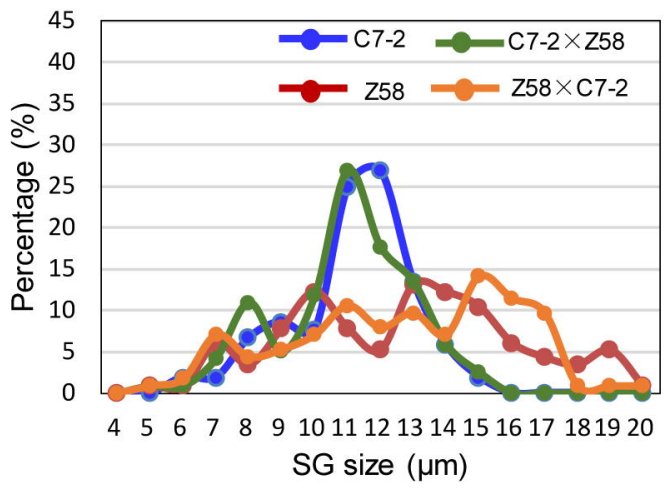

$100(\%)$ 
a
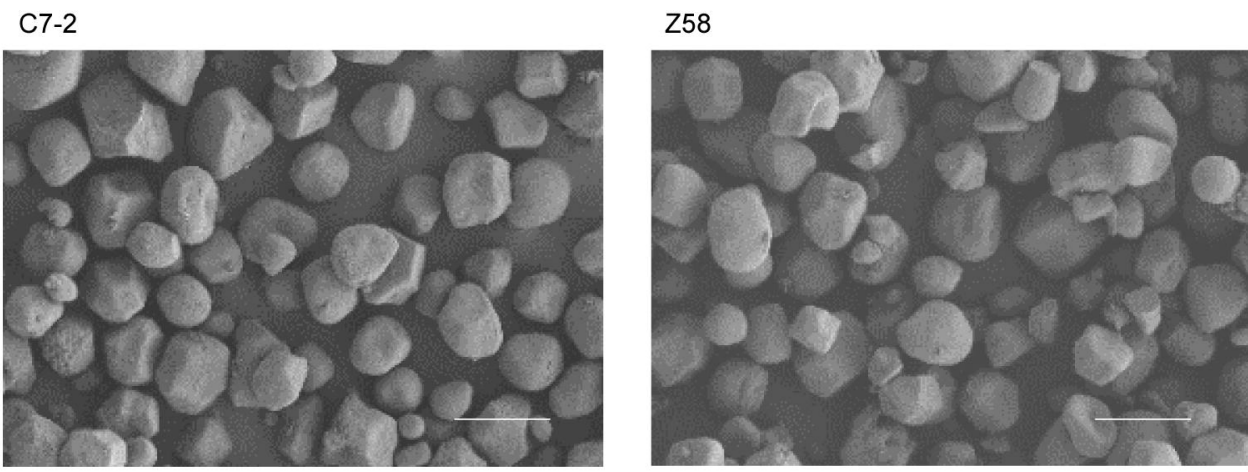

\section{C7-2×Z58}
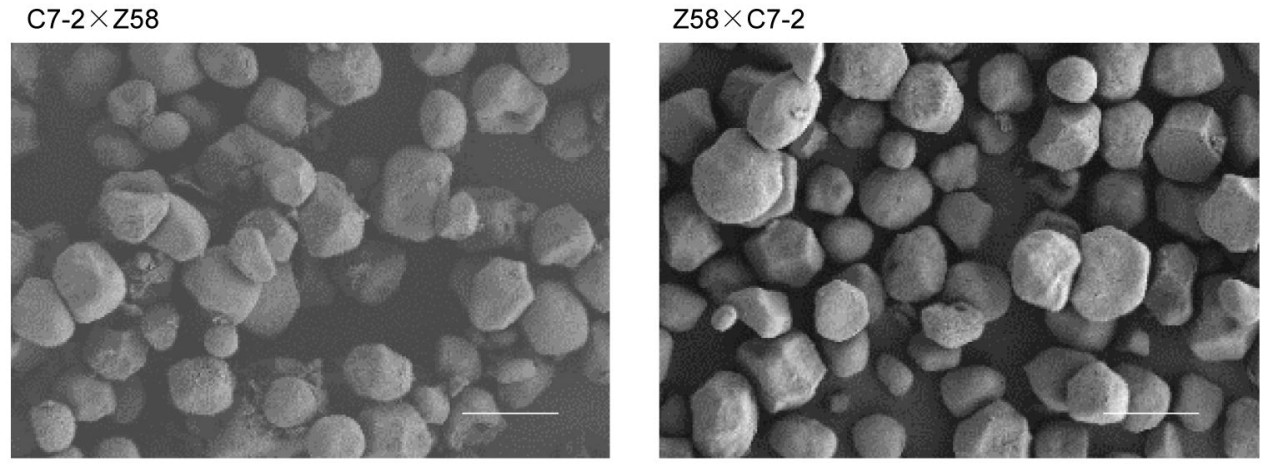

b

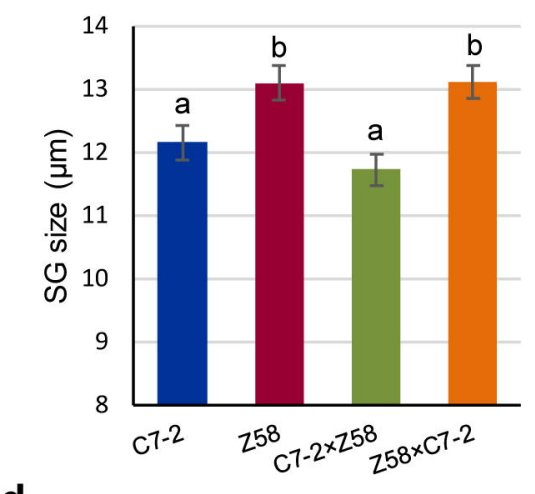

d

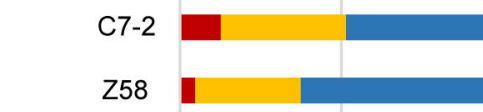

C7-2 $\times$ Z58

$\mathrm{Z} 58 \times \mathrm{C} 7-2$
C

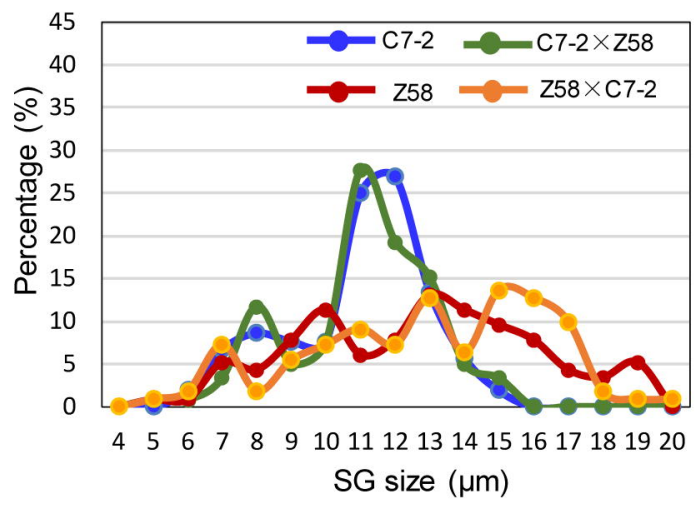

$\square<8 \mu \mathrm{m}$

$8 \mu \mathrm{m}-12 \mu \mathrm{m}$

$\square>12 \mu \mathrm{m}$ 